
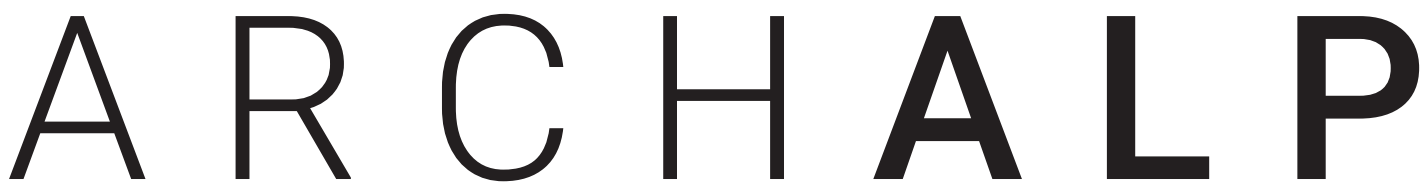

Rivista internazionale di architettura e paesaggio alpino / Revue internationale d'architecture et de paysage dans les Alpes / Internationale Zeitschrift für Alpine Architektur und Landschaft / Revija za alpsko arhitekturo in pokrajino / International journal of alpine architecture and landscape

Rer una nuota abitabilita delle Alpi.

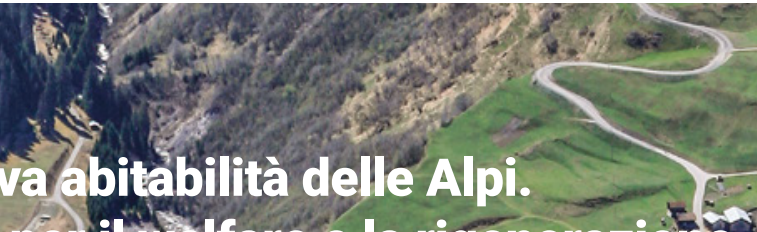
Architeture pet ilf lfare e la rigenerazione For a new inhabifability of the Alps. Architectures for welfare ând regeneration $/$ Pour une nouvelle habitabilité des Alpes. Architectures pour le welfare et la régéhérationy Für eine núue Bewohnbarkeit in den Alpen. Architekturen für Wohf ahrt und Regeneration / Za novo. bivalnost v Alpah, arhitekture za dobrobit in regeneracijo
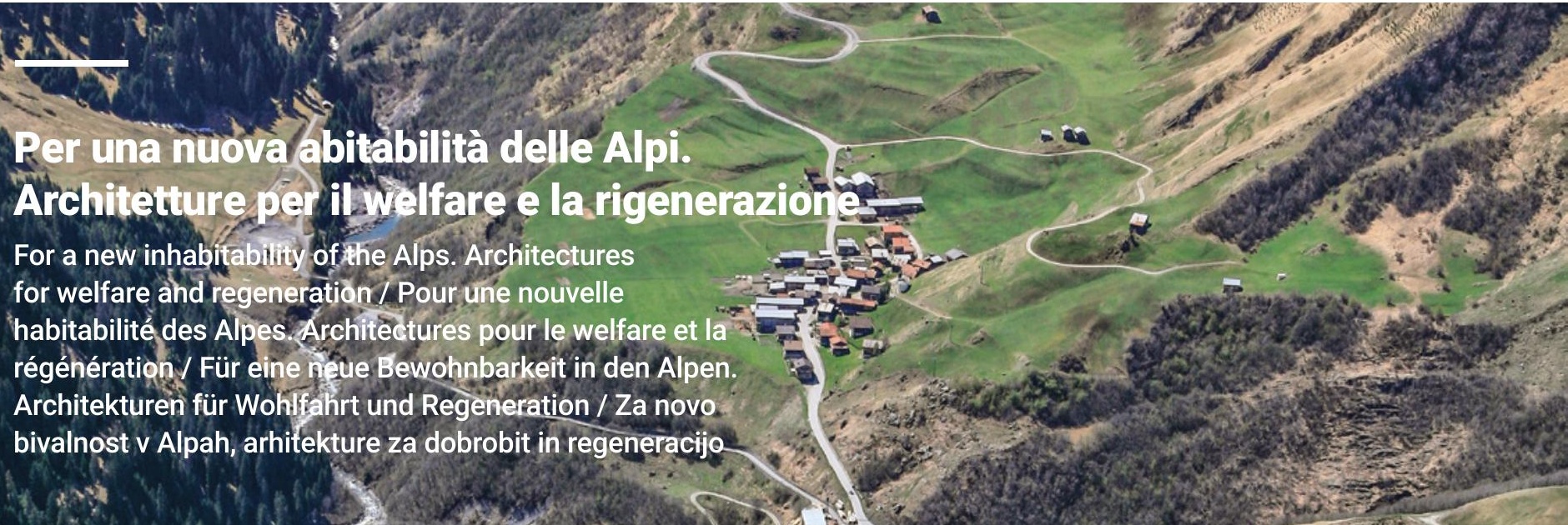
a $x \rightarrow 3$
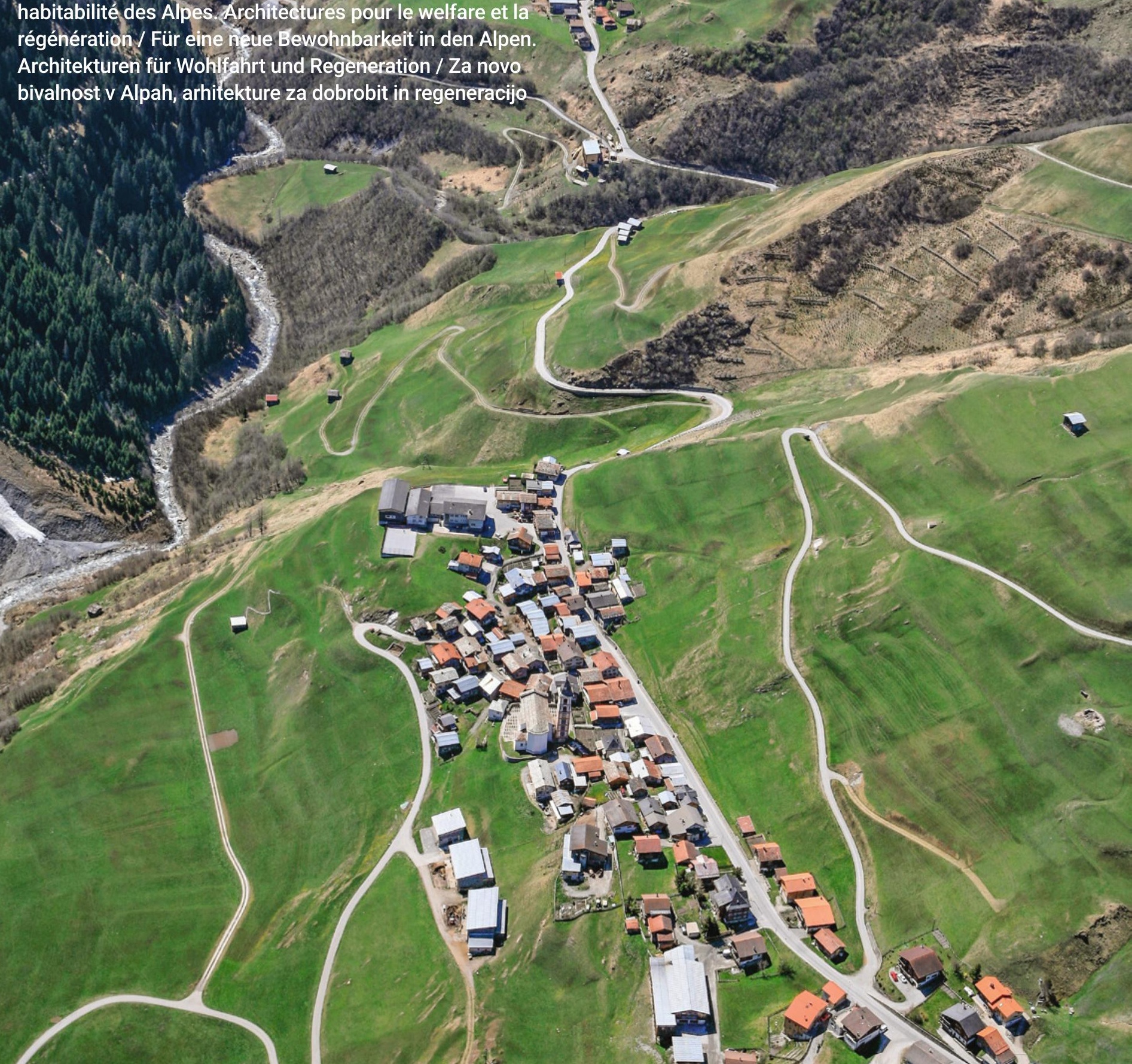


\section{Indice dei contenuti Contents}

\section{Temi}

Sulla centralità di spazio e territorio nel progetto di rigenerazione delle montagne e delle aree interne / On the centrality of space and territory in the project of regeneration of mountains and internal areas Antonio De Rossi, Laura Mascino

I servizi nelle Alpi italiane: quali e dove? Idee per uno scenario post-pandemico / Facilities in the Italian Alps: which ones and where? Ideas for a post-pandemic scenario

Giuseppe Dematteis

Manifesto di Camaldoli per una nuova centralità della montagna

Alla ricerca della distanza perduta. Rigenerare luoghi, persone e immaginari del riabitare alpino / In search of the lost distance. Regenerating places, people and images related to Alpine reinhabitation

Filippo Barbera, Andrea Membretti

Futuro e rigenerazione

Marco Bussone

\section{Esperienze}

Dorferneuerung zwischen Erhalten und Gestalten / 
Valades ousitanes, architettura e rigenerazione / Valades ousitanes,

Ostana e Topolò: hardware, software e welfare nelle comunità di "ritorno" / Ostana and Topolò: hardware, software and welfare in "return" communities

Margherita Valcanover

"Senza mostrare i muscoli": i progetti di rigenerazione di Tao+C e AZL nel solco della "prosperosa società" della Cina contemporanea / "Without flexing one's muscles": the regeneration projects of Tao+C and AZL in the wake of the "prosperous society" of contemporary China Edoardo Bruno, Dalila Tondo

L'archipel Butor. Une régénération, par la culture, d'un village soumis à la métropolisation genevoise / The Butor archipelago. A regeneration, through culture, of a village subject to the metropolisation of Geneva Arnaud Dutheil

Pratiche e progettualità di rigenerazione e welfare: il "Premio triennale Giulio Andreolli - Fare paesaggio" / Actions and projects of regeneration and welfare: the "Premio triennale Giulio Andreolli - Fare paesaggio" Giorgio Tecilla

Architetture e strategie per il welfare. Il caso di Brunico in Val Pusteria / Welfare architectures and strategies. The Bruneck case in Val Pusteria

Eleonora Gabbarini

Arhitektura oživlja / Architecture revives Kristina Dešman, Maja Ivanič

Si Crans-Montana meurt. Soigner le corps malade d'une station / If Crans-Montana dies. Taking care of the ailing body of a tourist resort Patrick Giromini 

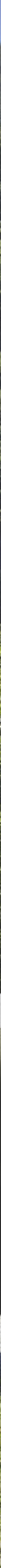


\title{
La costruzione dell'abitabilità in Val Bregaglia nel XX secolo
}

\author{
The construction of habitability in Val Bregaglia in the 20th century \\ Armando Ruinelli \\ edited by Anna Innocenti
}

\begin{abstract}
Both urbanized Alpine territories and cities share the need for a continuous renewal of spaces, and the theme of the regeneration of mountain areas is all the more topical due to the change in the ways of inhabiting such places. In order to play an active role in these transformations, contemporary architecture should take into account the interpretation of both the landscape and the urban fabric. Among the architect's analysis tools, comparison with the past plays a significant role, and especially in the Alpine valleys, where the circulation of ideas is sometimes slower or "overdue" compared to dynamic urban realities.

The occasional presence of professionals coming from other locations, often from cities, can be considered an opportunity to renew the local architectural culture; these architectures materialize perspectives "from an outside eye" and fresh interpretations of places.

In the Alpine valleys, tourism and the exploitation of water resources are two themes often related to the presence of "infiltrations": although Val Bregaglia is fairly untouched by tourism development, it provides some examples of holiday homes and bears the signs of large infrastructural interventions related to the exploitation of water resources.

During the twentieth century, there were no resident architects in Val Bregaglia. After the economic crisis of the years between the two world wars, design activities saw the intervention of architects such as Bruno Giacometti (Stampa, 1907-Zollikon, 2012), Peppo Brivio (Lugano 1923-2016), Tita Carloni (Rovio 1931-Mendrisio 2012) and Pierre Zoelly (Zurich 1923-2003).

More recently, Miller \& Maranta (Basel), H.J. Ruch (St. Moritz) and Lazzarini (Samedan) also carried out projects in this territory.
\end{abstract}

\section{Armando Ruinelli}

Born in Soglio in 1954, he is an FAS architect. In 2000 he founded the studio Ruinelli Associati Architetti SIA with Fernando Giovanoli.

\section{Anna Innocenti}

Born in Sodrio in 1985, she has been working as an architect at RA Ruinelli Associati Architetti since 2011.
Keywords

Val Bregaglia, heritage, regeneration, landscape, architecture, Alps.

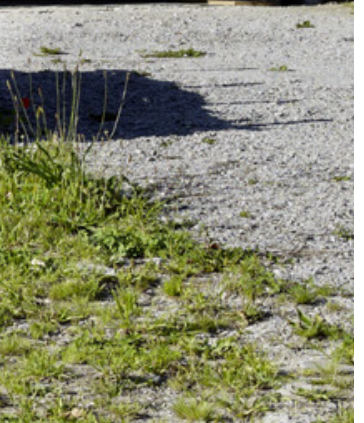


I territori alpini urbanizzati condividono con le città la necessità del continuo rinnovamento degli spazi. La rigenerazione perciò, sia essa relativa a una grande metropoli che a un piccolo nucleo, è un processo intrinseco e imprescindibile di ciò che è "urbano".

L'attualità del tema della rigenerazione dei nuclei montani è dovuta al cambiamento dei modi di abitare tali luoghi, con le nuove esigenze residenziali e la perdita - o ridimensionamento - di alcune funzioni, come quelle agricole, che ne hanno per lungo tempo caratterizzato la struttura insediativa, paesaggistica e sociale.

L'architettura contemporanea, per poter assumere un ruolo attivo in queste trasformazioni e una responsabilità nel mantenimento del valore dei nuclei, deve porsi la questione della lettura del paesaggio e del tessuto urbano. Fra gli strumenti di analisi dell'architetto vi è il confronto con gli edifici progettati da altri che hanno operato nel passato o operano tutt'ora all'interno di un territorio. In particolare nelle valli alpine, dove talvolta la circolazione di idee è più lenta o avviene "in ritardo" rispetto alle realtà urbane dinamiche, la conoscenza delle opere notevoli presenti e il dialogo con i loro autori non può che essere utile.

Si può considerare opportunità di rinnovamento della cultura architettonica locale la presenza occasionale di professionisti provenienti da altri luoghi, spesso cittadini, che hanno lasciato nei territori alpini degli edifici emblematici.

Tali architetture materializzano sguardi "da fuori" e interpretazioni dei luoghi offrendo spunti ai professionisti locali per rinnovare il loro stesso punto di vista sui "propri" territori.

Attraverso questi edifici può avvenire una sorta di "infiltrazione" di idee innovative, riguardo a morfologia, tipologia e tecniche costruttive, che può favorire la rigenerazione urbana.

Evidentemente il fatto di "venire da fuori" non è garanzia di migliori capacità dei progettisti, né di buona qualità delle opere.

Tuttavia, in presenza di opere architettonicamente valide, si può innescare uno stimolo positivo per i progettisti autoctoni e la cultura locale diffusa.
Nelle valli alpine due fattori in particolare hanno impresso nel corso del XX secolo una accelerazione alla trasformazione dei territori: il turismo e lo sfruttamento delle risorse idriche. Entrambi procedono secondo ritmi non lineari, a fasi di grande sviluppo seguono fasi di rallentamento, e richiamano interessi economici da fuori, più che da iniziative locali.

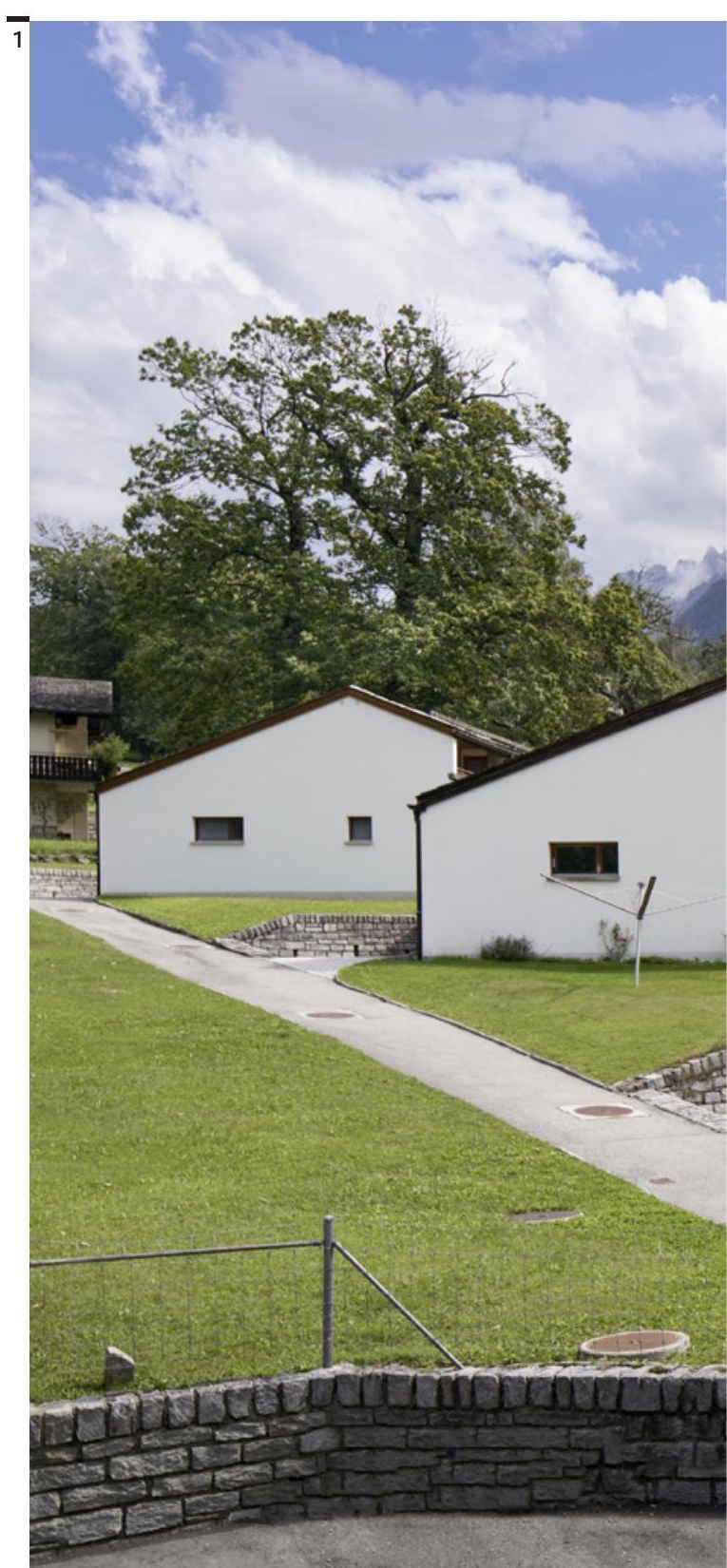


La presenza delle "infiltrazioni” è spesso connessa a questi due temi.

Anche la Val Bregaglia presenta questo scenario, pur essendo stata poco toccata dallo sviluppo turistico, ha degli esempi di case per vacanze (si veda Pierre Zoelly e Tita Carloni). Sul suo territorio ci sono testimonianze importanti di sfruttamento delle risorse idriche che hanno reso necessari grandi interventi infrastrutturali, opere più che altro ingegneristiche, ma che hanno introdotto in luoghi marginali i più avanzati progressi nella tecnica delle costruzioni e di sperimentazione dei materiali (si veda iniziativa città di Zurigo).

Fig. 1 Nel XX secolo in Val Bregaglia non esistono archiQuartiere Brentan a Castasegna, Bruno Giacometti, 19571959 (foto Ralph Feiner). tetti residenti. L'unica eccezione è Ottavio Ganzoni (Promontogno, 1873-1963), il quale, dopo aver studiato a Monaco di Baviera, rientra in valle $\mathrm{e}$ realizza, in giovane età e in breve tempo, i pro- getti più importanti dell'epoca: l'ospedale (Spino, 1902), l'albergo Elvezia (Vicosoprano, 1903), le scuole (Maloja, 1904 e Bondo, 1905), la villa Baldini (Orden-Maloja, 1906).

Negli anni tra le due guerre una grave crisi economica ferma qualsiasi attività costruttiva, in seguito alla quale l'edilizia privata viene praticata in maniera quasi esclusiva dagli impresari-costruttori locali. Le case che sorgono tra gli anni Cinquanta e la fine degli anni Settanta sono testimonianze di una prassi che non interpreta il preesistente, né la tradizione cosiddetta "vernacolare", né tantomeno le nuove istanze dell'architettura contemporanea. Le uniche opere pubbliche dell'epoca sono legate al progetto di sfruttamento idrico dell'Azienda della città di Zurigo, che inizia con la costruzione della diga dell'Albigna (1955-'61). I professionisti coinvolti sono perlopiù ingegneri e si occupano

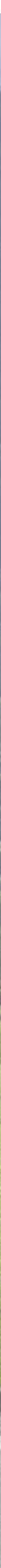


Fig. 2

Casa unifamiliare a Castasegna, cucina,

Ruinelli Associati Architetti, 2012-2013 (foto Ralph Feiner).

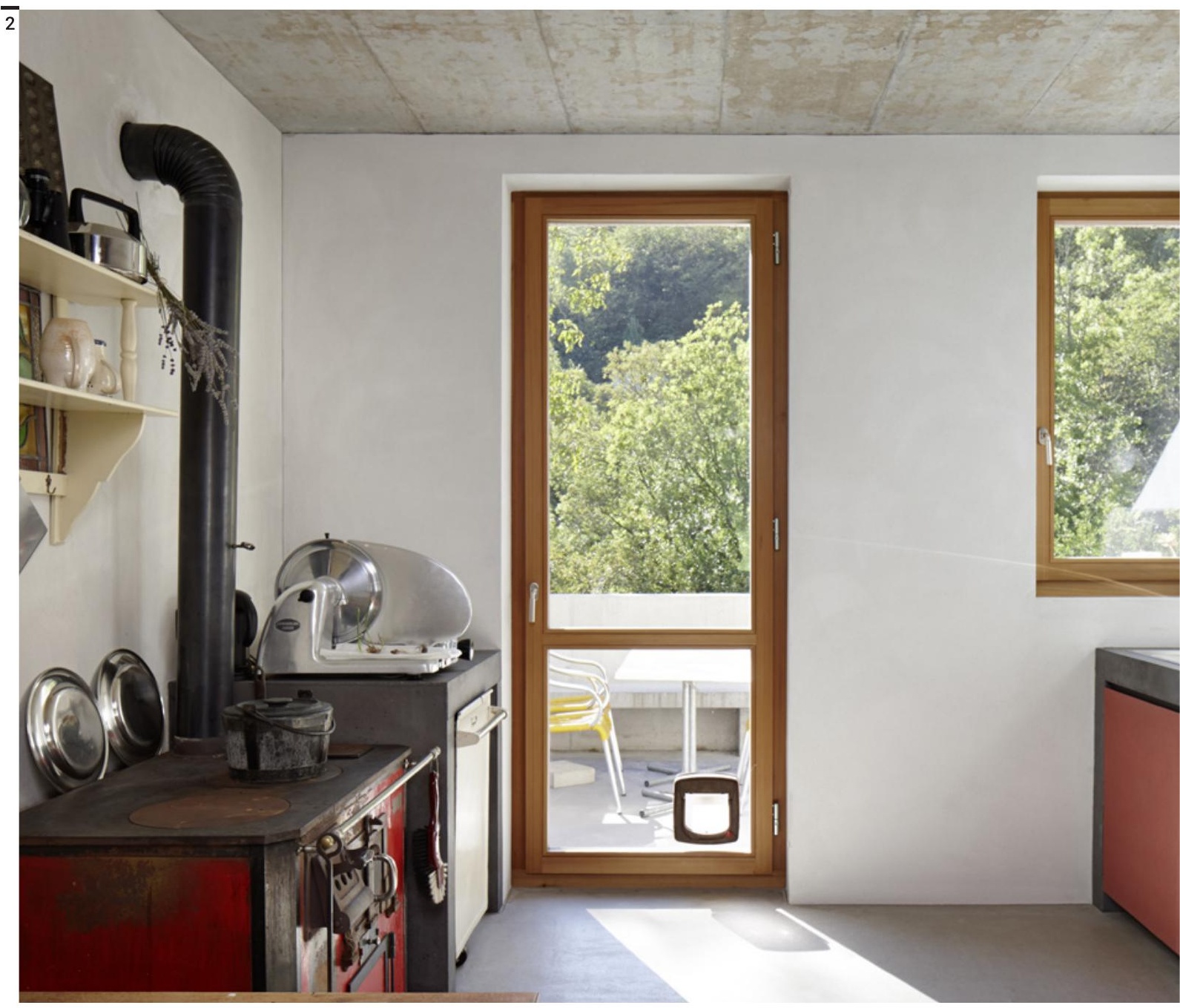

Fig. 3

Casa unifamiliare a

Castasegna, vista

est, Ruinelli Associati Architetti, 2012-2013

(foto Ralph Feiner).

Fig. 4

Casa unifamiliare a Castasegna, pianta piano terra, Ruinelli Associati Architetti, 2012-2013

Fig. 5 Casa unifamiliare a Castasegna, pianta primo piano, Ruinelli Associati Architetti, 2012-2013.

Fig. 6 Casa unifamiliare a Castasegna, sezione longitudinale, Ruinelli Associati Architetti, 2012-2013.

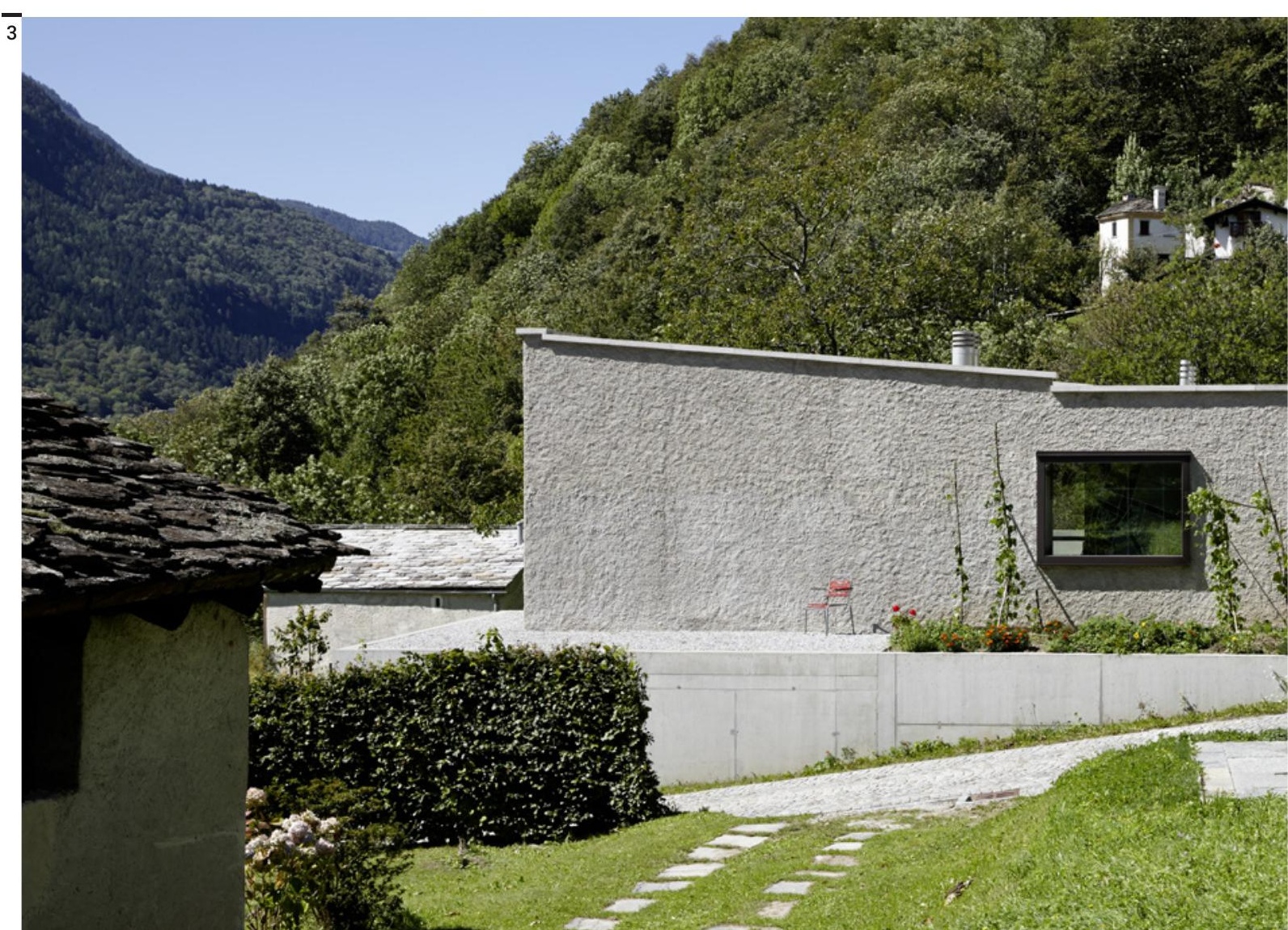



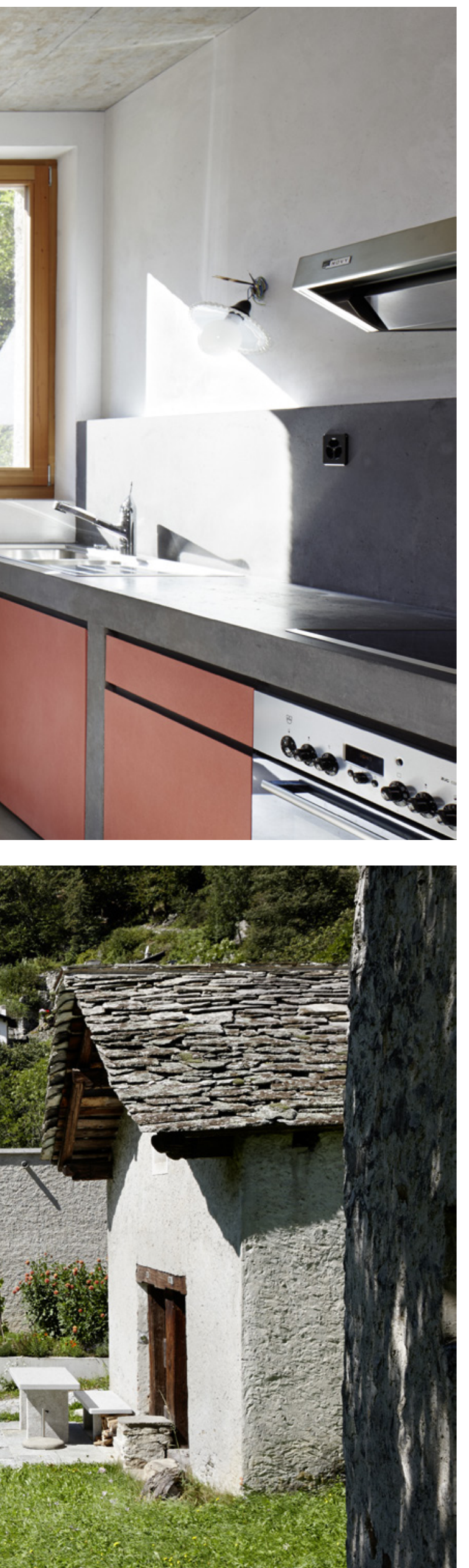

$\overline{4}$

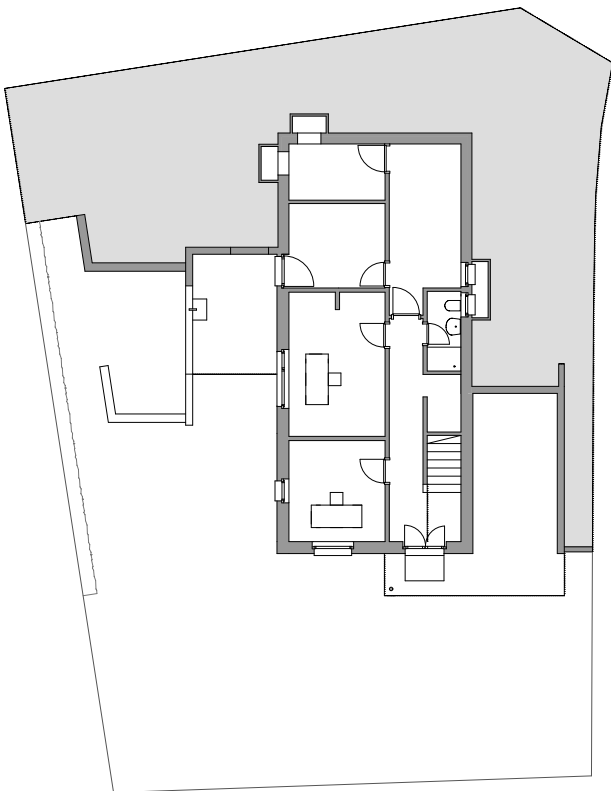

$\overline{5}$

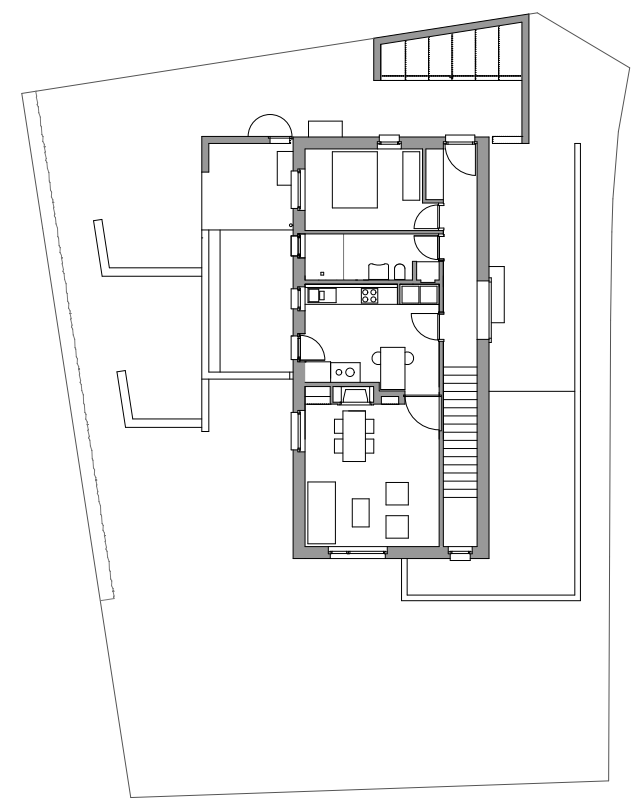

$\overline{6}$

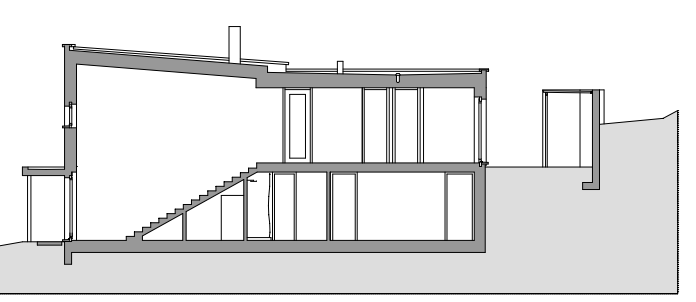


di infrastrutture che hanno un impatto importante a livello paesaggistico, ma meno significativo nelle ricadute sulla cultura architettonica locale. Tra questi progetti è tuttavia degno di nota l'edificio con la sala macchine di Löbbia, presso Casaccia, degli zurighesi Kurt e Hans Pfister.

Parallelamente alla realizzazione di queste opere, la stessa committenza individua in Bruno Giacometti (Stampa, 1907-Zollikon, 2012), un bregagliotto emigrato a Zurigo, la figura ideale per realizzare una serie di interventi architettonici e urbanistici esemplari.

Nella seconda metà del Novecento, Giacometti progetta numerose tra le più importanti opere pubbliche del periodo: l'edificio postale (Maloja, 1951), la stazione della teleferica Pranzaira-Albigna (Casaccia, 1954-'55), il casello per le dogane (Castasegna, 1955), il quartiere per impiegati delle forze idriche a San Cassiano (Vicosoprano, 1955), due case bifamiliari per gli impiegati doganali (Castasegna, 1955-'56), le scuole comunali a San Cassiano (Vicosoprano, 1956-'63), il quartiere residenziale Brentan (Castasegna, 1957-'59), la scuola comunale a Somarovan (Stampa, 1962), l'ampliamento dell'ospedale di Circolo a Flin (Spino, 1955, 1962-'63, 1975-'77).

L'attività di Giacometti in Bregaglia può essere letta come una emblematica declinazione del Movimento Moderno in area alpina. Se da una parte il suo lavoro rivela dimensioni e proporzioni che tendono verso la standardizzazione ripresa dal Modulor di Le Corbusier (si vedano per esempio le piante del quartiere Brentan), dall'altra la sensibilità verso i luoghi gli permette di gestire ogni incarico in maniera specifica, senza rifarsi a un canone formale prefissato. Giacometti riesce a combinare il linguaggio moderno con i materiali della tradizione, il rispetto delle preesistenze senza adattare anacronisticamente la sua architettura alle vecchie case, l'inserimento di nuovi edifici in contesti naturali da preservare collegando con naturalezza spazio interno ed esterno.

Il quartiere Brentan esprime un modello ancora attuale di insediamento residenziale in area alpina. Esso sorge nel castagneto che da Castasegna si estende fino a Soglio, in una zona con alcune radure, stalle e cascine, considerate patrimonio da proteggere. Le 12 case unifamiliari, tutte uguali, su due piani, sono distribuite in modo libero nelle radure preesistenti secondo uno stesso orientamento sul pendio che ne permette una lettura unitaria dell'insediamento.

Fig. 7 La pianta esprime l'idea di continuità tra interno

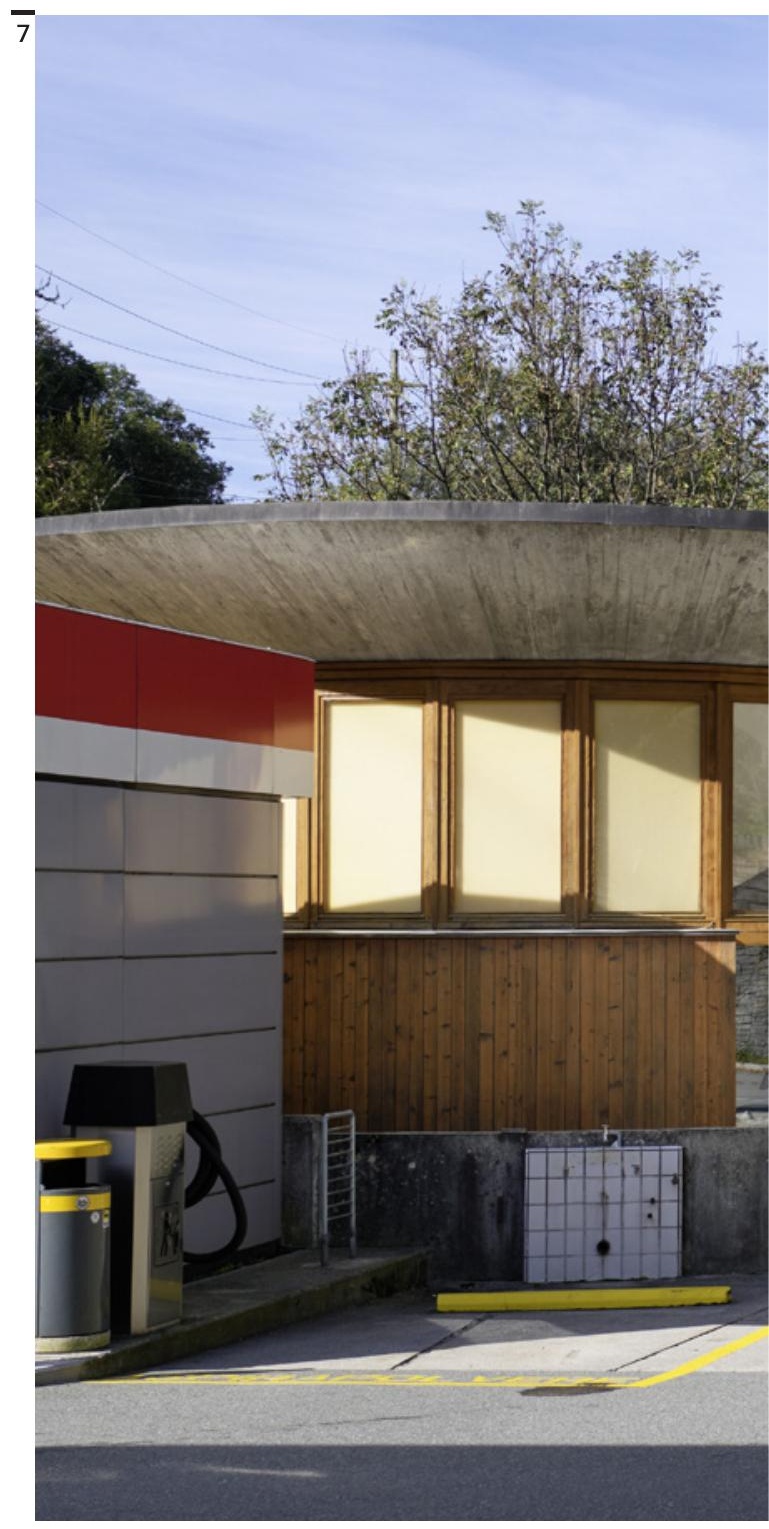

"Soprattutto nelle mie costruzioni in Bregaglia, i materiali edili - pietra naturale e legno - erano presenti, per così dire, in modo naturale sul cantiere. Utilizzarli, lo consideravo quasi ovvio, senza peraltro cadere in una architettura folcloristica. Ho sempre ritenuto essenziale l'adattamento in scala alla zona circostante, ma senza prendere in considerazione l'idea di un adattamento formale: ogni edificio dovrebbe esprimere le conoscenze e le funzioni della propria epoca" (AA.VV., 2008).

Anche nella scuola a Stampa Giacometti propone i medesimi materiali. In questo caso la pietra è particolarmente importante nell'avvicinare l'edificio alla natura. Il tetto a padiglione ricoperto in piode e i muri in sasso che sono presenti dentro e fuori l'edificio richiamano i mucchi di pietrame ai bordi dei terreni agricoli. In questo caso è perciò un elemento del paesaggio ad ispirare il con- 


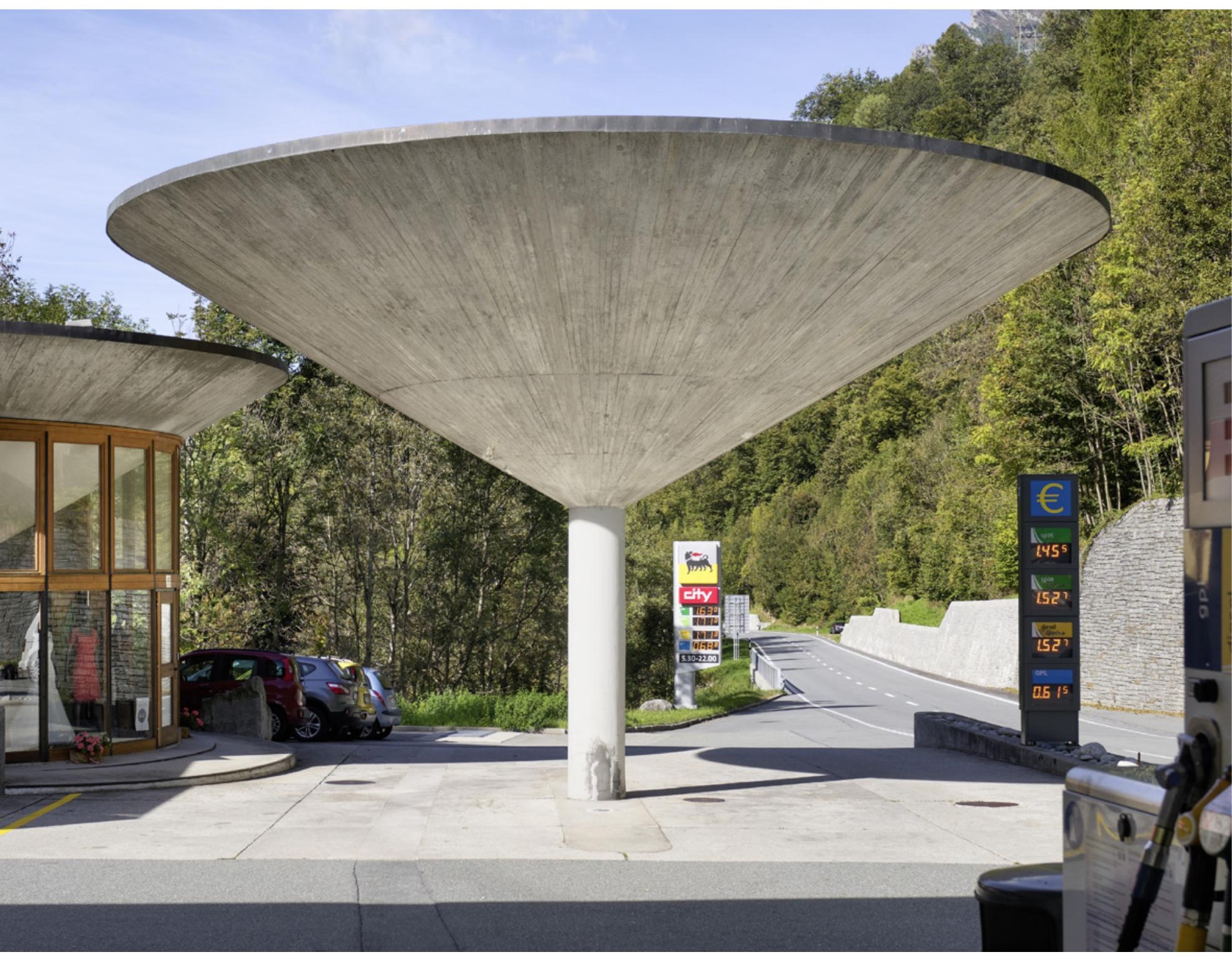

cetto di edificio solitario che sorge di fronte al villaggio di Stampa.

Nel secondo Novecento, oltre a Giacometti, altri architetti non autoctoni intervengono in Bregaglia, i più significativi sono: Peppo Brivio (Lugano 1923-2016) con la stazione di rifornimento Farzett (Castasegna, 1962), Tita Carloni (Rovio 1931-Mendrisio 2012) con la casa di vacanza (Maloja, 1970) e Pierre Zoelly (Zurigo 1923-2003) con la casa di vacanza (Stampa, 1978).

Più recentemente, hanno operato gli architetti Miller\&Maranta (Basilea) con il Roccolo (Castasegna, 2003-2004), H. J. Ruch (St. Moritz) con la casa-rifugio (Roticcio, 2014) e Lazzarini (Samedan) con la mensa a Somarovan (Stampa, inizio anni 2000) e l'albergo Longhin (Maloja, 2015).

La stazione con distributori e chioschi di Peppo Brivio esprime una architettura radicale e spe- rimentale. Tre "funghi", o "alberi", in cemento a vista dal diametro di $10,80 \mathrm{~m}$, dallo spessore decrescente, poggiano su un esile pilastro cavo al cui interno sono convogliate le acque raccolte dal grande ombrello rovesciato. I serramenti dei due chioschi hanno telai in larice molto sottili che si raccordano in modo elegante alla struttura di cemento.

Qui l'architettura è ridotta al massimo, con un risultato di grande forza espressiva. Oggi tale radicalità sarebbe difficile da raggiungere poiché le istanze della fisica della costruzione sono spesso preponderanti rispetto alle tematiche architettoniche e non concedono più la possibilità di usare profili così sottili, né di "dimenticarsi" degli strati di isolamento.

La casa per vacanze progettata da Pierre Zoelly è altro esempio di "infiltrazione" interessante da 
commentare, poiché compie due operazioni: da una parte si pone come una casa nuova che reinterpreta la tipologia della stalla, intento che oggi lascia dei dubbi sia per quanto riguarda il concetto che per la sua espressione nelle proporzioni del fronte principale. Allo stesso tempo, Zoelly inserisce elementi che non hanno niente a che vedere con il tema della stalla, come il camino centrale, e materiali attuali e sperimentali per l'epoca, come il compensato negli interni e la lamiera per le ante delle finestre. L'esperimento è interessante perché gioca su due campi, il contemporaneo e il recupero di un passato lontano.

È significativo notare che 15 anni dopo la stazione di Peppo Brivio lo slancio verso una architettura radicale sia da Zoelly sostituito con una architettura più simbolica, tesa verso il recupero di elementi del passato - il tema della stalla - che, se interpretati con la concettualizzazione e sensibilità attuale, sollevano delle perplessità.

Due esempi contemporanei, il depuratore comunale presso Stampa e la centrale idroelettrica di Villa di Chiavenna, sembrano declinare i temi dell'architettura simbolica, tuttavia banalizzando elementi formali che vengono utilizzati per camuffare la reale funzione dell'edificio, forse considerata non degna di espressione.

Il rivestimento in legno del depuratore è infatti inappropriato, tende a far sembrare l'edificio qualcosa che non è. Così come la centrale idroelettrica che "somiglia" a una grande stalla, fa finta di essere una architettura d'altri tempi, ma nelle proporzioni completamente diverse - e legate alla sua funzione - rivela al secondo sguardo la sua natura e l'incongruenza della veste architettonica.

Un rapporto con le opere "alpine" di Bruno Giacometti sembra essere invece più presente nella casa unifamiliare di Castasegna, progettata dal nostro studio nel 2012-2013.

L'edificio interpreta i temi del paesaggio, in particolare il pendio su cui sorge, ai margini del castagneto. La casa segue il terreno, creando una molteplicità di spazi aperti differenziati e su più livelli, con una varietà di collegamenti tra interno ed esterno. Il tetto nel suo fronte principale è in contropendenza rispetto al pendio, abbassandosi dal prospetto principale sud verso la linea di impluvio della falda. L'innalzamento della falda conferisce un effetto slanciato all'edificio, che è compatto, lungo e stretto.

Il tema del muro è qui molto significativo, a partire dall'impostazione costruttiva della casa che

Fig. 8

Scuola comunale a Stampa, Bruno

Giacometti, 1962

(foto Ralph Feiner). de anch'esso il tema del muro, così come il coronamento dell'edificio, un muro coperto da elementi prefabbricati in cemento che richiamano i tradizionali muri degli orti intonacati con copertura in pietra.

La casa ha una morfologia e un linguaggio autonomi rispetto al luogo, per esempio abbandona la "regola" del tetto a falde, tuttavia risulta integrata nel contesto, grazie alle proporzioni e alla materializzazione delle facciate. La composizione dei fronti è risolta con bucature in formati diversi, tra loro legati da rapporti proporzionali. La superficie degli esterni è ottenuta con intonaco monostrato in mal-

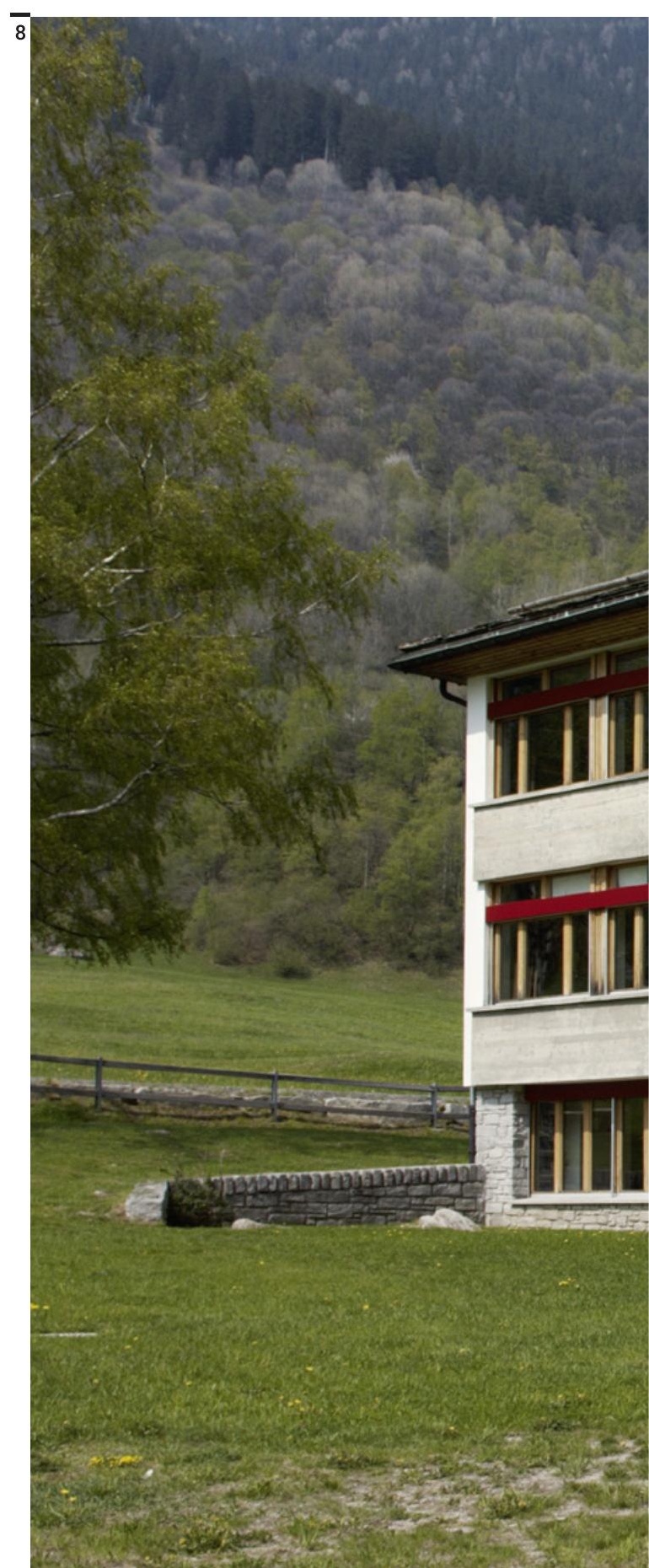


ta di calce, cemento e ghiaietto, applicato e "lavato" a mano.

All'interno, la lunga scala e il corridoio permettono di leggere l'edificio in tutta la sua lunghezza e altezza. Le solette sono in calcestruzzo a vista e i pavimenti in betoncino autolivellante, finito a olio, a mano dagli stessi proprietari. La cucina è concepi- ta come un "laboratorio" e realizzata in calcestruzzo nero, gettato sul posto.

Si tratta di una casa costruita con poche risorse e materiali utilizzati al grezzo. Tuttavia il budget ridotto non ha compromesso né la qualità architettonica né la ricerca compositiva, compresi il progetto del dettaglio e la cura artigianale.

\section{Bibliografia}

AA.VV. (2008), «Bruno Giacometti architetto», in Quaderni grigionitaliani, n. 2, monografico.

Obrist Robert, Semadeni Silva, Giovanoli Diego (1986), Construir/ Bauen/ Costruire 1830-1980, Verlag Werk AG.

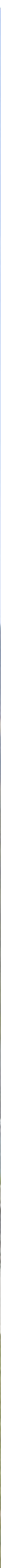

